\title{
QFLan: A Tool for the Quantitative Analysis of Highly Reconfigurable Systems
}

\author{
Vandin, Andrea; Ter Beek, Maurice H.; Legay, Axel; Lluch Lafuente, Alberto
}

Published in:

Formal Methods

Link to article, DOI:

10.1007/978-3-319-95582-7_19

Publication date:

2018

Document Version

Peer reviewed version

Link back to DTU Orbit

Citation (APA):

Vandin, A., Ter Beek, M. H., Legay, A., \& Lluch Lafuente, A. (2018). QFLan: A Tool for the Quantitative Analysis of Highly Reconfigurable Systems. In K. H., J. P., B. R., \& E. D. V. (Eds.), Formal Methods (pp. 329-337). Springer. Lecture Notes in Computer Science Vol. $10951 \mathrm{https} / / /$ doi.org/10.1007/978-3-319-95582-7 19

\section{General rights}

Copyright and moral rights for the publications made accessible in the public portal are retained by the authors and/or other copyright owners and it is a condition of accessing publications that users recognise and abide by the legal requirements associated with these rights.

- Users may download and print one copy of any publication from the public portal for the purpose of private study or research.

- You may not further distribute the material or use it for any profit-making activity or commercial gain

- You may freely distribute the URL identifying the publication in the public portal 


\title{
QFLan: A Tool for the Quantitative Analysis of Highly Reconfigurable Systems
}

\author{
Andrea Vandin ${ }^{1(凶)}$, Maurice H. ter Beek ${ }^{2}$, \\ Axel Legay $^{3}$, and Alberto Lluch Lafuente ${ }^{1}$ \\ 1 DTU, Lyngby, Denmark \\ \{anvan, albl\}@dtu.dk \\ 2 ISTI-CNR, Pisa, Italy \\ maurice.terbeek@isti.cnr.it \\ 3 Inria, Rennes, France \\ axel.legay@inria.fr
}

\begin{abstract}
QFLAN offers modeling and analysis of highly reconfigurable systems, like product lines, which are characterized by combinatorially many system variants (or products) that can be obtained via different combinations of installed features. The tool offers a modern integrated development environment for the homonym probabilistic feature-oriented language. QFLAN allows the specification of a family of products in terms of a feature model with quantitative attributes, which defines the valid feature combinations, and probabilistic behavior subject to quantitative constraints. The language's behavioral part enables dynamic installation, removal and replacement of features. QFLAN has a discrete-time Markov chain semantics, permitting quantitative analyses. Thanks to a seamless integration with the statistical model checker MultiVeStA, it allows for analyses like the likelihood of specific behavior or the expected average value of non-functional aspects related to feature attributes.
\end{abstract}

\section{Introduction}

Product line engineering is a methodology that aims to develop and manage, in a cost-effective and time-efficient manner, a family of products or (re)configurable system variants, to allow the mass customization of individual variants. Their variability is captured by feature models, whose features represent stakeholderrelevant functionalities or system aspects [1]. The challenge when lifting successful modeling and analysis techniques for single systems to families of products or configurable systems, is to handle their variability, due to which the number of possible variants may be exponential in the number of features. This led to so-called family-based analyses [32]: analyse properties on an entire product line and use variability knowledge about valid feature configurations to deduce results for individual products. This is applied in, e.g., $8,10,11,15,19,23,28,30$.

In $\sqrt{2} \sqrt{4}$, we presented various facets of the probabilistic modeling language QFLAN, capable of describing a wide spectrum of aspects of (software) product lines (SPL). The type of quantitative constraints that are supported by QFLAN are significantly more complex than those commonly associated to 
attributed feature models $[9,18,26]$. This paper presents the QFLAN tool, a multi-platform tool for the specification and analysis of QFLAN models, which has been implemented in the Eclipse environment using XTEXT technology, thus obtaining a state-of-the-art integrated development environment (IDE). The tool is available, together with installation and usage instructions, from http://github.com/qflanTeam/QFLan.

Related work The QFLAN prototypes from [2,3] were the first tools that offered statistical model checking tailored for SPL, generating approximately correct results via sampling, which is particularly useful on very large models when exact model checking is infeasible 25. Next to dedicated exact model checkers such as VMC [6] and the tool suite ProVeLines [17], which offers the best known SPL-specific model checker, SNIP [12, also popular model checkers like mCRL2 and SPIN have been made amenable to SPL model checking $|7| 8|19| 21$. Furthermore, the tool ProFeat [11] extends the probabilistic model checker PRISM [24] with feature-oriented concepts to be able to model families of stochastic systems and to analyze them through probabilistic model checking. QFLAN scales to larger models with respect to precise probabilistic analysis techniques. In fact, we can handle (cf. http://github.com/qflanTeam/QFLan and [5]) significantly larger instances of the Elevator case study. Originally introduced in [29], this case study is now a benchmark for SPL analysis known to be very demanding in terms of scalability when large sizes of Elevator systems are considered (cf., e.g., [5, 10, 11, 13, 14, 18, 19]).

Outline Sect.2 describes the tool's architecture, while Sect. 3 applies the tool to a simple family of coffee vending machines. Sect. 4 concludes the paper.

\section{QFLan Architecture}

The architecture of QFLAN is sketched in Fig. 1. It consists of a GUI layer and a core layer, devoted to modeling and analysis tasks, respectively.

GUI layer The components of the GUI layer are depicted in Fig. 2. The QFLAN editor provides state-of-the-art editing support, including auto-completion, syntax and error highlighting, and fix suggestions. This was obtained using XTEXT technology. For instance, the editor does on-the-fly error-detection on the structure

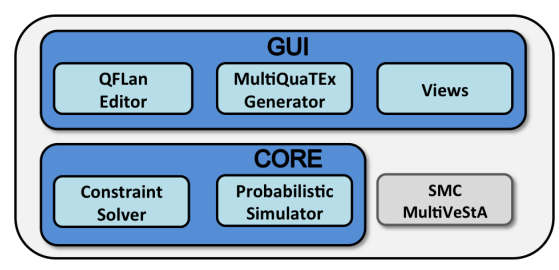

Fig. 1. QFLAN's architecture of the feature model. The editor also offers support for MultiQuaTEx, the query language of the statistical model checker MultiVeStA [31] integrated in the tool. In particular, the user can specify properties to be analyzed with a high-level language consisting of QFLAN ingredients only, from which MultiQuaTEx queries are automatically generated. In addition, the GUI layer offers a number of views, including the project explorer to navigate across different QFLAN models, the tree-like outline to navigate in the elements of QFLAN model, the console view to display diagnostic information, and the plot view to display analysis results. 


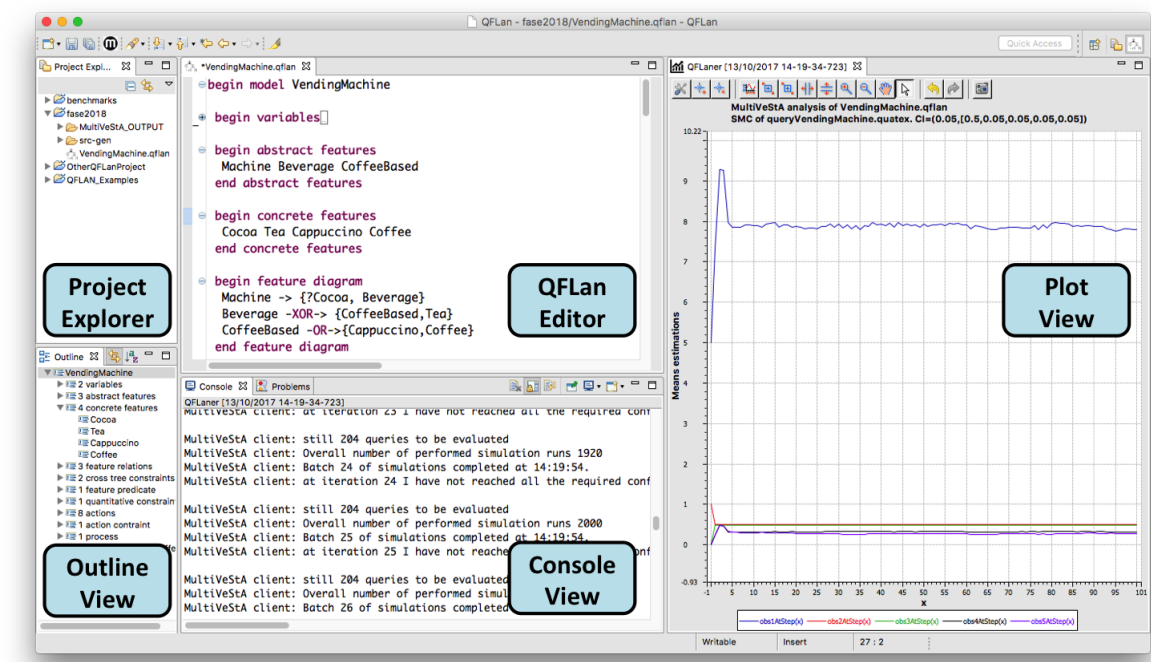

Fig. 2. A screenshot of the QFLAN tool

Core layer The main component of the core layer is a probabilistic simulator. According to QFLAN's semantics, each state can have a set of outgoing admissible transitions, each labeled with a weight to compute the probability distribution of the transitions outgoing from each state. This leads to a discretetime Markov chain semantics. Starting from the initial configuration specified by the modeler, the simulator iteratively computes all one-step transitions allowed in the state, and probabilistically selects one according to the probability distribution that results from normalizing the rates of the generated transitions. In particular, to check whether a transition is admissible the tool uses an ad-hoc constraint solver to guarantee that the transition does not violate any of the constraints specified by the modeler.

In 3. 4], we presented a prototypical implementation of a QFLAN simulator based on the Maude toolkit [16] and Microsoft's SMT solver Z3 [27], integrated with MultiVeStA. The mature QFLAN tool presented in this paper has been redeveloped from scratch using Java-based technologies in order to obtain a multi-platform modern IDE for QFLAN instead of a command-line prototype. Furthermore, this led to an analysis speedup of several orders of magnitude.

\section{QFLan at Work: Coffee Vending Machine}

We consider a family of vending machines inspired by examples from the literature (e.g., $2,60,8,15,28]$ ). For illustration purposes, we consider a simple version. Larger case studies can be found at http://github.com/qflanTeam/QFLan, including the bike-sharing case study used as running example in $[2 \mid 4]$, the above mentioned SPL benchmark Elevator case study used in [5] to evaluate QFLAN's scalability, and a case study concerning risk analysis of a safe lock system with variability used in [5] to illustrate QFLAN's applicability in a non-SPL setting. 
This family of vending machines sells either tea, or the coffee-based beverages coffee, cappuccino, and cappuccino with cocoa (chocaccino). Its feature model is depicted in Fig. 3. Listing 1 shows its QFLAN specification in 1:1 correspondence.

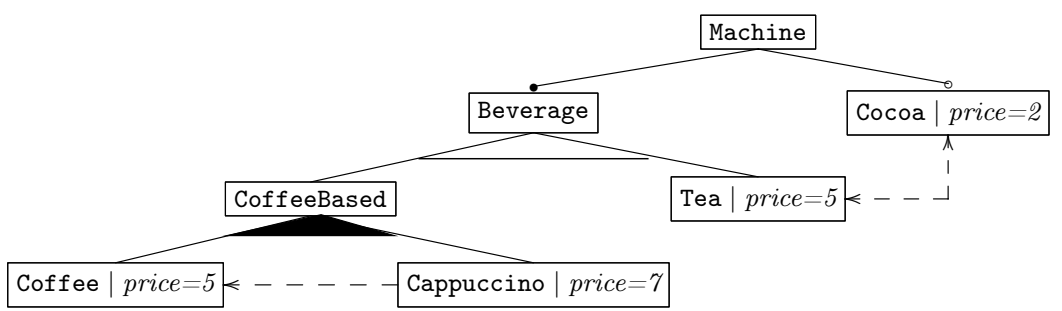

Fig. 3. Feature model of vending machine product line

Each node is a feature, while edges denote constraints defining admissible combinations of installed features. As is common in feature models, we distinguish between concrete and abstract features. The former are the tree's leaves, and can be explicitly (un)installed, whereas the latter are internal nodes, used mainly to group features. The root denotes a product, i.e. a specific vending machine. To instantiate a machine, one may install the optional feature Cocoa (its optionality is denoted by a circle in Fig. 3 and with a ? in Listing 1), while it must contain the mandatory feature Beverage (as denoted by a filled circle in Fig. 3 and by the absence of a ? in Listing 11. Finer constraints on the presence of features other than mandatory or optional also can be imposed. The machine may come equipped with either a Tea dispenser or with one for CoffeeBased beverages. This is specified by the XOR edges connecting Beverage to Tea and to CoffeeBased. The CoffeeBased dispenser can be used to pour Coffee, Cappuccino, or both, as denoted by the OR edges.

Features can also be subject to cross-tree constraints. The arrow from Cappuccino to Coffee denotes that the former requires the latter, the rationale being that coffee is a prerequisite for preparing cappuccino. Instead, the double-headed arrow connecting Cocoa and Tea denotes that they exclude each other, since cocoa only serves to prepare chocaccino. Such constraints are specified in QFLAN as shown in Lines 15,18 of Listing 1. Finally, features can have quantitative predicates as attributes. In the example, all concrete features have price as attribute (Lines 20 22 of Listing 1). Abstract features implicitly inherit all predicates from concrete ones, with the cumulative value of all descendant concrete features actually installed.

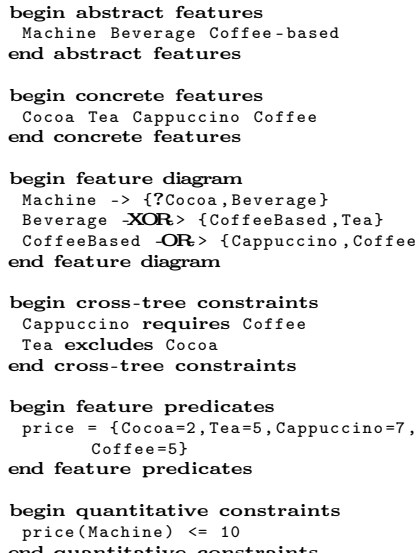

Listing 1. QFLAN encoding of feature model displayed in Fig. 3 
Lines 24 26 of Listing 1 show that QFLAN supports another family of constraints regarding feature predicates, the quantitative constraints, used in this case study to exclude machines with a cumulative price that is superior to 10 .

The dynamics of our example family is sketched in Fig. 4, while Listing 2 shows a textual QFLAN specification in close correspondence. The machine, initialized with a Coffee dispenser only, is pre-configured in the FACTORY by possibly installing any admissible feature configuration. After this, one can sell the machine to a company, in whose DEPOSIT minor customizations can be done before deployment. Once in OPERATION, the machine can serve customer requests, depending on the installed features, or be reconfigured in the DEPOSIT.

In QFLAN, one first specifies real-valued variables (Lines 13 of Listing 2) that can be used in the guards of constraints or to facilitate the analysis phase. Variables can be updated as side effects of the execution of actions, defined in the actions block (Lines 5 8). In addition, also installed concrete features can be executed as actions, meaning a user is using them. Note that Chocaccino is not a feature (like Cappuccino or Coffee) but an action. The rationale is that any machine provided with Cappuccino and Cocoa dispensers can serve Chocaccino. This is expressed in QFLAN using yet another family of constraints, the action constraints (Lines 10 12). Finally, Lines 1432 specify the actual behavior in terms of a process named dynamics with sets of states (Line 15) and transitions (Lines 16 31), corresponding to the nodes and edges, respectively, of Fig. 4 . Each transition is labeled with an action, including the (un)installation or replacement of features, and its weight, used to calculate the probability that it is executed.

The model is completed by specifying an initial configuration (Lines 3437 ).

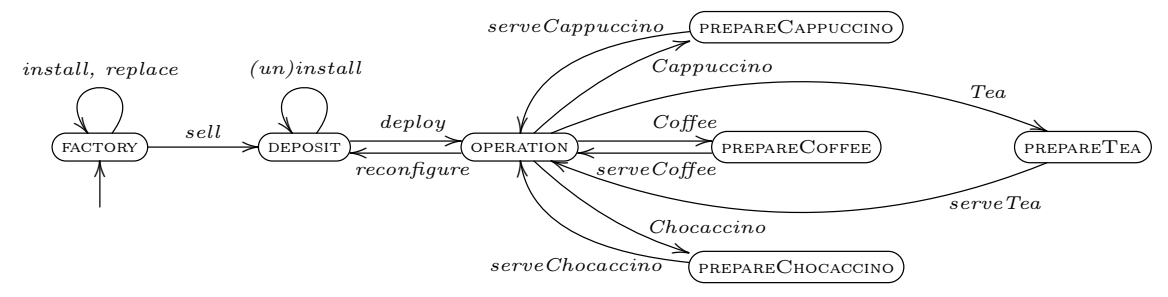

Fig. 4. Sketch of vending machine behavior

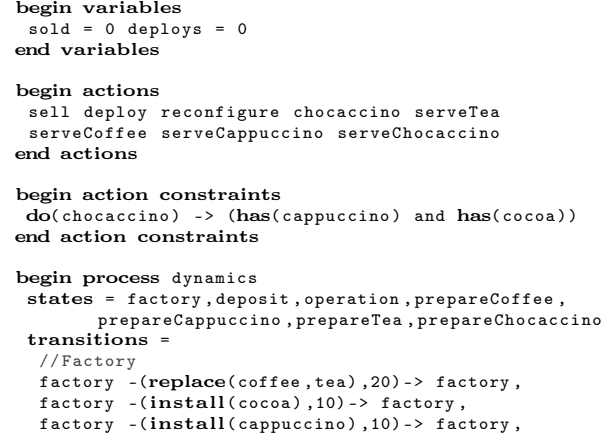

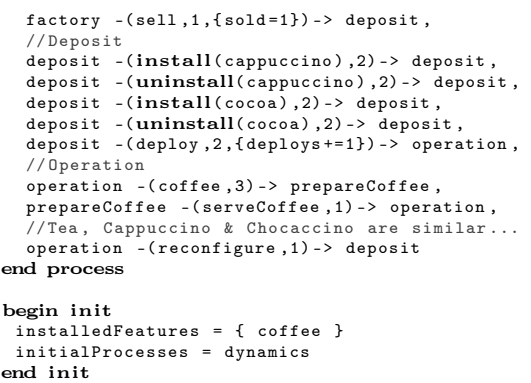

Listing 2. QFLAN encoding of vending machine behavior sketched in Fig. 4 
Note that, as side-effect of executing an action (sell), the transition in Line 20 sets the variable sold to 1 when a machine is sold. Pinpointing this precise moment allows to study, e.g., the average price of sold machines, or the probability that they have dispensers for Coffee, Tea, Cappuccino, and Cocoa.

These five properties (average price and four distinct probabilities) can be expressed as in Listing 3. The query specifies the properties to evaluate in the first state that satisfies $\operatorname{sold}==1$. The expected value $x$ of each property is estimated by MultiVeStA as the mean value $\bar{x}$ of $n$ samples

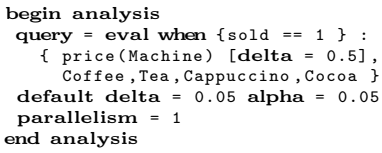

Listing 3. QFLAN properties (obtained from $n$ independent simulations), with $n$ large enough such that with probability $(1-\alpha)$ we have $x \in[\bar{x}-\delta / 2, \bar{x}+\delta / 2]$. Default values of $\alpha$ and $\delta$ are provided by the user via the keywords alpha and delta (these can be overruled for a specific property by providing its new value in square brackets, cf. Line 3). Finally, keyword parallelism $=$ p allows to distribute simulations across p processes to be allocated on different cores. The analysis of the five properties in Listing 3 with QFLAN required \pm 2000 simulations, for which it ran in under a second on a laptop with a $2.4 \mathrm{GHz}$ Intel Core i5 processor and $4 \mathrm{~GB}$ of RAM.

The probability of having Cappuccino dispensers in machines sold is zero: any machine always has at least either a Tea or Coffee dispenser, with price 5, so installing Cappuccino would violate the constraint price (Machine) $<=10$ because Cappuccino has price 7 . For the same reason, the probability to have Coffee installed is 0.33 , which is roughly $\frac{1+10}{1+10+20}$, since in Lines 1820 of Listing 2 we see that 1,10 , and 20 are the weights assigned to sell, install(Cocoa), and replace (Coffee, Tea), respectively (the weight of installing Cappuccino is ignored as it is not allowed). Note that Coffee is installed in machines sold if replace (Coffee,Tea) is not executed, which happens if sell is executed first, or if Cocoa gets installed, which prevents the execution of replace (Coffee, Tea) due to constraint Tea excludes Cocoa. The probability of installing Cappuccino becomes 0.46 if we use the more permissive constraint price (Machine) $<=15$.

QFLAN also allows to study parametric properties as time progresses. For example, by replacing "when $\{$ sold $==1.0\}$ " with "from 0 to 100 by 1 " in Listing 3 , we study the five properties at each of the first 100 simulation steps, for a total of 500 properties. Analysis results of parametric properties are visualized in interactive plots, in this case the one in Fig. 2, computed in a few seconds.

\section{Outlook}

We presented QFLAN, a quantitative modeling and verification environment for highly (re)configurable systems, like SPL, including Eclipse-based tool support. QFLAN offers an IDE for specifying system configurations and their probabilistic behavior in a high-level language as well as advanced statistical analyses of nonfunctional properties based on a discrete-time Markov chain semantics.

In the future, we envision a stochastic semantics based on continuous-time Markov chains for the analysis of time-related properties and a semantics based on featured transition systems to interface with the ProVeLines tool suite [17]. 


\section{References}

1. Apel, S., Batory, D.S., Kästner, C., Saake, G.: Feature-Oriented Software Product Lines: Concepts and Implementation. Springer (2013). https://doi.org/10.1007/978-3-642-37521-7

2. ter Beek, M.H., Legay, A., Lluch Lafuente, A., Vandin, A.: Quantitative Analysis of Probabilistic Models of Software Product Lines with Statistical Model Checking. In: FMSPLE 2015. EPTCS, vol. 182, pp. 56-70 (2015). https://doi.org/10.4204/EPTCS.182.5

3. ter Beek, M.H., Legay, A., Lluch Lafuente, A., Vandin, A.: Statistical Analysis of Probabilistic Models of Software Product Lines with Quantitative Constraints. In: SPLC 2015. pp. 11-15. ACM (2015). https://doi.org/10.1145/2791060.2791087

4. ter Beek, M.H., Legay, A., Lluch Lafuente, A., Vandin, A.: Statistical Model Checking for Product Lines. In: ISoLA 2016. LNCS, vol. 9952, pp. 114-133 (2016). https://doi.org/10.1007/978-3-319-47166-2_8

5. ter Beek, M.H., Legay, A., Lluch Lafuente, A., Vandin, A.: A framework for quantitative modeling and analysis of highly (re)configurable systems (2018), http://arxiv.org/abs/1707.08411

6. ter Beek, M.H., Mazzanti, F., Sulova, A.: VMC: A Tool for Product Variability Analysis. In: FM 2012. LNCS, vol. 7436, pp. 450-454 (2012). https://doi.org/10.1007/978-3-642-32759-9 36

7. ter Beek, M.H., de Vink, E.P.: Using mCRL2 for the Analysis of Software Product Lines. In: FormaliSE 2014. pp. 31-37. ACM (2014). https://doi.org/10.1145/2593489.2593493

8. ter Beek, M.H., de Vink, E.P., Willemse, T.A.C.: Family-Based Model Checking with mCRL2. In: FASE 2017. LNCS, vol. 10202, pp. 387-405 (2017). https://doi.org/10.1007/978-3-662-54494-5_23

9. Benavides, D., Segura, S., Ruiz-Cortés, A.: Automated analysis of feature models 20 years later: A literature review. Inf. Syst. 35(6), 615-636 (2010). https://doi.org/10.1016/j.is.2010.01.001

10. Chrszon, P., Dubslaff, C., Klüppelholz, S., Baier, C.: Family-based modeling and analysis for probabilistic systems - featuring ProFeAt. In: FASE 2016. LNCS, vol. 9633, pp. 287-304 (2016). https://doi.org/10.1007/978-3-662-49665-7_17

11. Chrszon, P., Dubslaff, C., Klüppelholz, S., Baier, C.: ProFeat: feature-oriented engineering for family-based probabilistic model checking. Formal Asp. Comput. 30(1), 45-75 (2018). https://doi.org/10.1007/s00165-017-0432-4

12. Classen, A., Cordy, M., Heymans, P., Legay, A., Schobbens, P.Y.: Model checking software product lines with SNIP. Int. J. Softw. Tools Technol. Transf. 14(5), 589-612 (2012). https://doi.org/10.1007/s10009-012-0234-1

13. Classen, A., Cordy, M., Heymans, P., Legay, A., Schobbens, P.Y.: Formal semantics, modular specification, and symbolic verification of product-line behaviour. Sci. Comput. Program. 80(B), 416-439 (2014). https://doi.org/10.1145/2499777.2499781

14. Classen, A., Heymans, P., Schobbens, P.Y., Legay, A.: Symbolic model checking of software product lines. In: ICSE 2011. pp. 321-330. ACM (2011). https://doi.org/10.1145/1985793.1985838

15. Classen, A., Heymans, P., Schobbens, P.Y., Legay, A., Raskin, J.F.: Model Checking Lots of Systems: Efficient Verification of Temporal Properties in Software Product Lines. In: ICSE 2010. pp. 335-344. ACM (2010). https://doi.org/10.1145/1806799.1806850 
16. Clavel, M., Durán, F., Eker, S., Lincoln, P., Martí-Oliet, N., Meseguer, J., Talcott, C. (eds.): All About Maude-A High-Performance Logical Framework: How to Specify, Program and Verify Systems in Rewriting Logic, LNCS, vol. 4350 (2007). https://doi.org/10.1007/978-3-540-71999-1

17. Cordy, M., Classen, A., Heymans, P., Schobbens, P.Y., Legay, A.: ProVeLines: A Product Line of Verifiers for Software Product Lines. In: SPLC 2013. pp. 141-146. ACM (2013). https://doi.org/10.1145/2499777.2499781

18. Cordy, M., Schobbens, P.Y., Heymans, P., Legay, A.: Beyond Boolean Product-Line Model Checking: Dealing with Feature Attributes and Multi-features. In: ICSE 2013. pp. 472-481. IEEE (2013). https://doi.org/10.1109/ICSE.2013.6606593

19. Dimovski, A.S., Al-Sibahi, A.S., Brabrand, C., Wąsowski, A.: Efficient family-based model checking via variability abstractions. Int. J. Softw. Tools Technol. Transf. 19(5), 585-603 (2017). https://doi.org/10.1007/s10009-016-0425-2

20. Dimovski, A.S., Al-Sibahi, A.S., Brabrand, C., Wąsowski, A.: Family-Based Model Checking Without a Family-Based Model Checker. In: SPIN 2015. LNCS, vol. 9232, pp. 282-299 (2015). https://doi.org/10.1007/978-3-319-23404-5_18

21. Dimovski, A.S., Wąsowski, A.: Variability-Specific Abstraction Refinement for Family-Based Model Checking. In: FASE 2017. LNCS, vol. 10202, pp. 406-423 (2017). https://doi.org/10.1007/978-3-662-54494-5 24

22. Gruler, A., Leucker, M., Scheidemann, K.D.: Modeling and Model Checking Software Product Lines. In: FMOODS 2008. LNCS, vol. 5051, pp. 113-131 (2008). https://doi.org/10.1007/978-3-540-68863-1 8

23. Kowal, M., Schaefer, I., Tribastone, M.: Family-Based Performance Analysis of Variant-Rich Software Systems. In: FASE 2014. LNCS, vol. 8411, pp. 94-108 (2014). https://doi.org/10.1007/978-3-642-54804-8 7

24. Kwiatkowska, M., Norman, G., Parker, D.: PRISM 4.0: Verification of Probabilistic Real-Time Systems. In: CAV 2011. LNCS, vol. 6806, pp. 585-591 (2011). https://doi.org/10.1007/978-3-642-22110-1_47

25. Legay, A., Delahaye, B., Bensalem, S.: Statistical Model Checking: An Overview. In: RV 2010. LNCS, vol. 6418, pp. 122-135 (2010). https://doi.org/10.1007/978-3642-16612-9_11

26. Mauro, J., Nieke, M., Seidl, C., Yu, I.C.: Context Aware Reconfiguration in Software Product Lines. In: VaMoS 2016. pp. 41-48. ACM (2016). https://doi.org/10.1145/2866614.2866620

27. de Moura, L., Bjørner, N.: Z3: An efficient SMT solver. In: TACAS 2008. LNCS, vol. 4963, pp. 337-340 (2008). https://doi.org/10.1007/978-3-540-78800-3 24

28. Muschevici, R., Proença, J., Clarke, D.: Feature Nets: behavioural modelling of software product lines. Softw. Sys. Model. 15(4), 1181-1206 (2016). https://doi.org/10.1007/s10270-015-0475-z

29. Plath, M., Ryan, M.: Feature integration using a feature construct. Sci. Comput. Program. 41(1), 53-84 (2001). https://doi.org/10.1016/S0167-6423(00)00018-6

30. Salay, R., Famelis, M., Rubin, J., Sandro, A.D., Chechik, M.: Lifting Model Transformations to Product Lines. In: ICSE 2014. pp. 117-128. ACM (2014). https://doi.org/10.1145/2568225.2568267

31. Sebastio, S., Vandin, A.: MultiVeStA: Statistical Model Checking for Discrete Event Simulators. In: ValueTools 2013. pp. 310-315. ACM (2013). https://doi.org/10.4108/icst.valuetools.2013.254377

32. Thüm, T., Apel, S., Kästner, C., Schaefer, I., Saake, G.: A Classification and Survey of Analysis Strategies for Software Product Lines. ACM Comput. Surv. 47(1), 6:1-6:45 (2014). https://doi.org/10.1145/2580950 